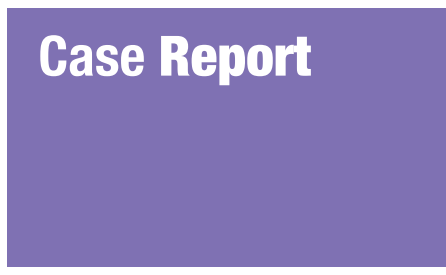

Submitted: 5 Mar 2016 Accepted: 13 June 2016 Online: 30 June 2016

\title{
Rare Presentation of Pseudomyxoma Retroperitonei: Stretching the Limits
}

\author{
Roumina HASAN ${ }^{1}$, Sandeep KUMAR ${ }^{2}$, Anuradha ck RAO ${ }^{3}$, \\ Rajagopal KADAVIGERE ${ }^{1}$
}

\author{
1 Department of Pathology, Melaka Manipal Medical College, Manipal \\ University, Manipal 576104, India \\ 2 Department of Radiology, Kasturba Medical College, Manipal University, \\ Manipal 576104, India \\ 3 Department of Pathology, Kasturba Medical College, Manipal University, \\ Manipal 576104, India
}

\begin{abstract}
To cite this article: Hasan R, Kumar S, Rao AC, Kadavigere R. Rare presentation of pseudomyxoma retroperitonei: stretching the limits. Malays J Med Sci. 2016; 23(4): 79-85. doi: 10.21315/mjms2016.23.4.11
\end{abstract}

To link to this article: http://dx.doi.org/10.21315/mjms2016.23.4.11

\begin{abstract}
A 55-year-old woman presented with a mucopurulent sinusal discharge from the right supragluteal region, with symptoms over the previous five months. This abscess began as a slowly swelling growth, which eventually turned into a discharging sinus, and she was diagnosed with a gluteal abscess. The patient underwent incisional drainage, and intra-operatively, the sinus tract could be seen extending to the retroperitoneum. A subsequent CT scan and an MRI of the abdomen revealed a large heterogeneous retroperitoneal cystic mass on the right side of midline, extending inferiorly into the anterior thigh along the iliopsoas. Superiorly, a tubular projection extended from the lesion, indenting the ileocaecal junction, while a fluid filled cutaneous fistulous tract was seen, extending to the right flank. A diagnosis of pseudomyxoma retroperitonei, likely of retrocaecal appendicular origin, was proposed. An explorative laparotomy with an appendectomy, and the evacuation of the retroperitoneal collection were completed. The subsequent histopathology confirmed the diagnosis of appendicular mucinous cystadenoma, with pseudomyxoma retroperitonei.
\end{abstract}

Keywords: pseudomyxoma retroperitonei, magnetic resonance imaging, appendix, cystadenoma, cystadenocarcinoma

\section{Introduction}

Pseudomyxoma retroperitonei (PMR) is a rare condition, characterised by a mucinous collection in the retroperitoneum, with only 45 documented cases having been reported in the English literature (1). The most common presentation of PMR is an abdominal mass with pain, while three cases have presented with spontaneous cutaneous fistulae (2-4), and only a single case exhibited a mass in the anterior thigh region (5).

Here, we report the unique presentation of a rare condition, in which a large retroperitoneal pseudomyxoma presented as a non-healing cutaneous sinus in right supragluteal region, masquerading as a gluteal abscess. Upon imaging, it was found to extend into the anterior thigh region. We will highlight the imaging characteristics of the PMR using contrast enhanced magnetic resonance imaging (MRI) and computed tomography (CT), and lay out the clinical and radiological approaches to such a unique case.

\section{Case History}

A 55-year-old-woman was admitted to the clinic with a 5-month history of a slow growing soft tissue swelling, which had turned into a discharging sinus in the right supragluteal region. The swelling was insidious upon onset, and had progressively increased in size. The patient had a history of a low grade, intermittent fever 
associated with chills, and had been followed up for a gluteal abscess. Previously, she had received various antibiotic treatments, but her condition kept deteriorating. Furthermore, she had no history of trauma, backache or bladder/bowel involvement. Upon examination, there was local erythema and increased warmth, with a $8 \times 6 \mathrm{~cm}$ freely mobile, tender, soft tissue swelling in her right flank. Granulation tissue and a purulent discharge were seen in the wound.

The systemic examination was otherwise normal, while the laboratory investigation revealed leucocytosis with a WBC count of $12.3 \times$ $10^{3} / \mu$, neutrophilia (neutrophils: $85 \%$ ), a raised ESR $(79 \mathrm{~mm} / \mathrm{hr}$ ) and raised $\mathrm{C}$ reactive protein (CRP: $214.4 \mathrm{mg} / \mathrm{L}$ ). The normal ranges for the WBC count, neutrophils, ESR and CRP being 4-10 $\times 10^{3} / \mu \mathrm{l}, 42-74 \%, \mathrm{O}-20 \mathrm{~mm} / \mathrm{hr}$ and $\mathrm{o}-6$ $\mathrm{mg} / \mathrm{L}$, respectively. The provisional diagnosis of a gluteal abscess was made, and incisional drainage under spinal anaesthesia was conducted. This revealed a large mucoid material filling a sinus tract extending from the right flank epidermis, deep into the retroperitoneal space. Additionally, $500 \mathrm{ml}$ of mucoid material was drained and sent for a histopathological examination.

An MRI of the abdomen was requested, and revealed a large ill-defined multiseptated retroperitoneal cystic lesion in the right lumbar and iliac fossal regions, extending inferiorly into the right anterior thigh. The lesion was hypointense in the T1-weighted images and hyperintense in the T2-weighted images, while superiorly, it showed a tubular projection abutting the ileocaecal junction (Figure 1). There was a fistulous tract extending from the mass lesion, superolaterally, just above the iliac bone, opening onto the skin in the right supragluteal region. Inferiorly, the mass was seen to be extending along the iliopsoas muscle, into the right anterior thigh, displacing the femoral vessels anteromedially (Figure 2). The psoas muscle was severely indented and effaced by the lesion; however, it appeared to be uninvolved in the disease process.

Post contrast, there was diffuse heterogeneous enhancement of the lesion, with enhancing peripheral and internal thick septations (Figure 3). The height and signal intensity of the lumbar vertebrae and intervertebral discs were normal. The CT scan revealed heterogeneously enhancing septae, of slightly higher than water attenuation, in the mucoid component of the retroperitoneal cystic lesion. Additionally, there was a thick walled tubular structure extending from the caecum, ending at the margin of the retroperitoneal collection (Figure 4). The right psoas muscle was indented by the retroperitoneal collection, with no erosion or destruction of the visualised pelvic bones or spine.

A provisional diagnosis of retroperitoneal pseudomyxoma, likely of retrocaecal appendicular origin, was suggested. A cancer antigen (CA)-125 test was conducted to determine the malignant potential of the pseudomyxoma, and it was found to be within normal limits at $26.2 \mathrm{U} / \mathrm{ml}$ (normal range: $\mathrm{O}-35 \mathrm{U} / \mathrm{ml}$ ).

An explorative laparotomy with an appendectomy and the evacuation of the pseudomyxomatous tissue were completed under general anaesthesia, where the appendix was found to be retrocaecal, and its ruptured tip was seen to be discharging mucoid material into the retroperitoneum. Both ovaries were found to be normal. The appendix and the mucoid material were sent for histopathology, which revealed an appendiceal mucinous cystadenoma, showing a focal low grade dysplasia, with a low grade pseudomyxoma retroperitonei (Figure 5).

The patient's post-operative recovery was uneventful, with regular dressings applied, and the drain was removed on the fifth postoperative day. No chemotherapy or radiotherapy was required, and during the 6-month follow up the patient was found to be disease free. 
Case Report | Pseudomyxoma retroperitonei stretching the limits

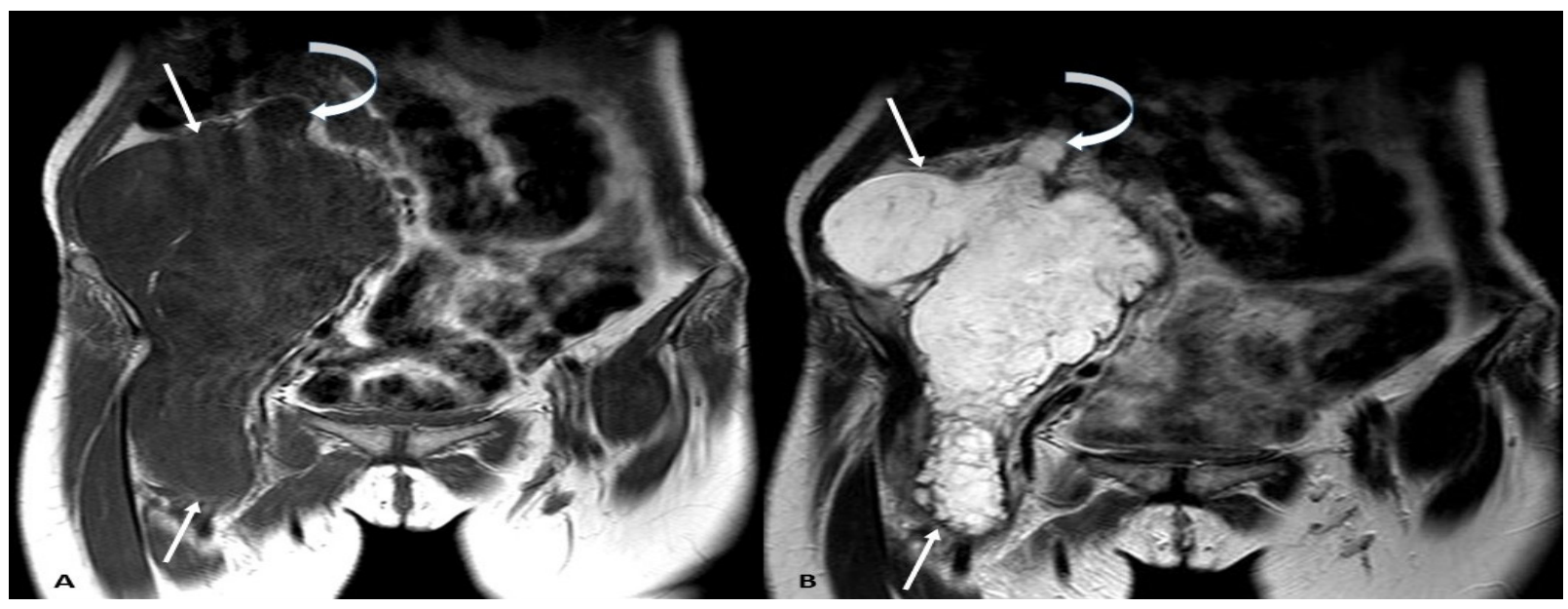

Figure 1: MRI coronal images reveal a large lobulated mass lesion in the right lumbar and iliac regions, extending inferiorly into the thigh (arrows), showing a tubular projection extending superiorly up to the ileocaecal region (curved arrow). It appears hypointense on T1-weighted (A) and intensely hyperintense on T2-weighted images (B).

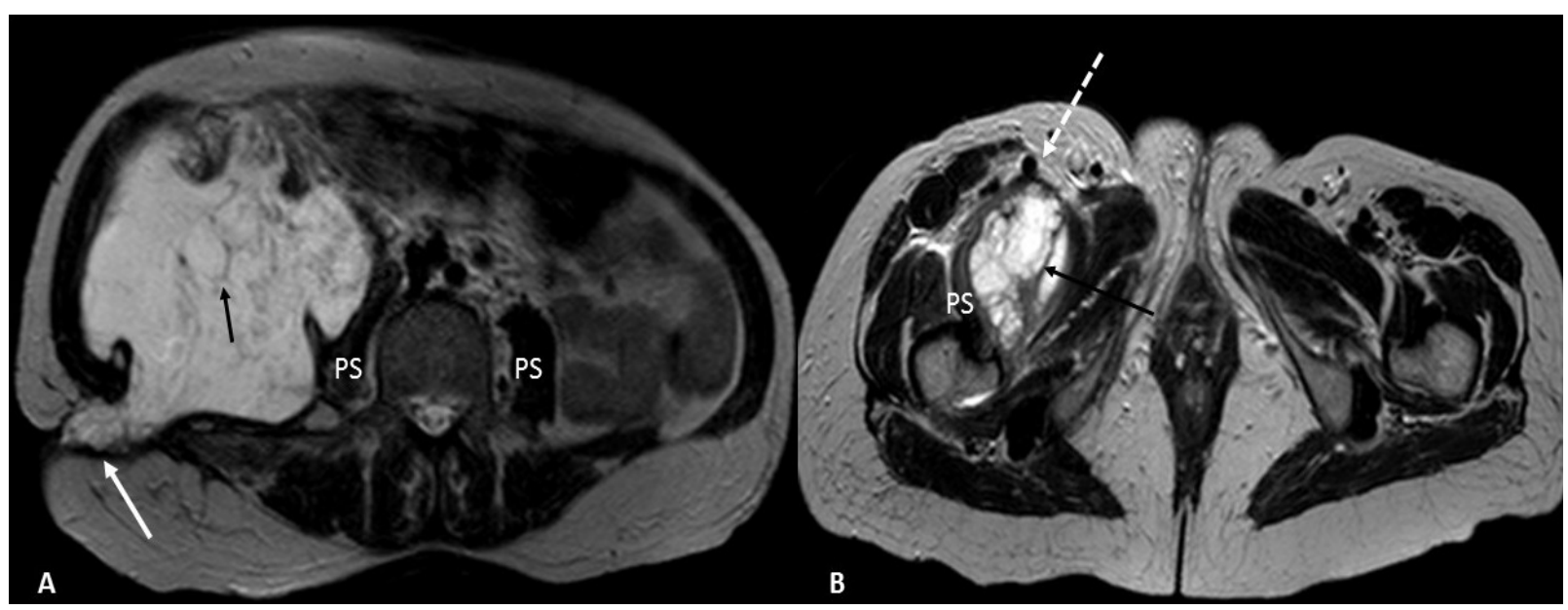

Figure 2: T2-weighted axial MRI images (A, B) reveal a fistulous tract extending posterolaterally from the retroperitoneal cystic lesion to the epidermis in the right flank region, just lateral to the quadratus lumborum (white arrow), medially indenting the right psoas muscle (PS). Inferiorly, the mass extends into the anterior thigh along the iliopsoas muscle, displacing the femoral vessels anteromedially (dashed arrow). Multiple thick hypointense internal septations are seen within the mass lesion (black arrows). 


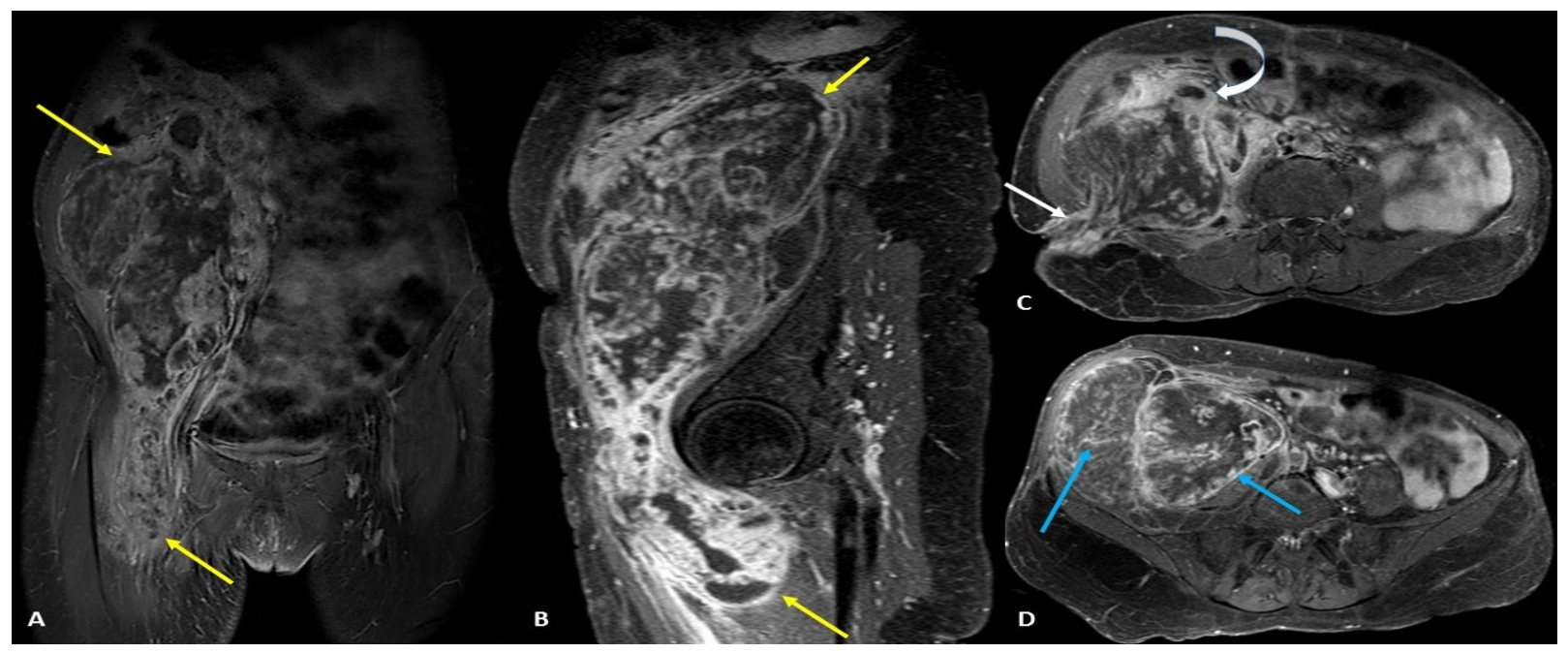

Figure 3: Post contrast T1 fat suppressed coronal and sagittal (A, B) images, revealing the cranio-caudal extension of the heterogeneously enhancing retroperitoneal cystic mass lesion, from the right lumbar region to the right anterior thigh (yellow arrows). The axial images (C, D) reveal the thick walled enhancing tubular structure closely abutting the collection superiorly (curved arrow), with enhancement along the fistulous tract (white arrow) in the peripheral rim, and within the internal septations (blue arrows).

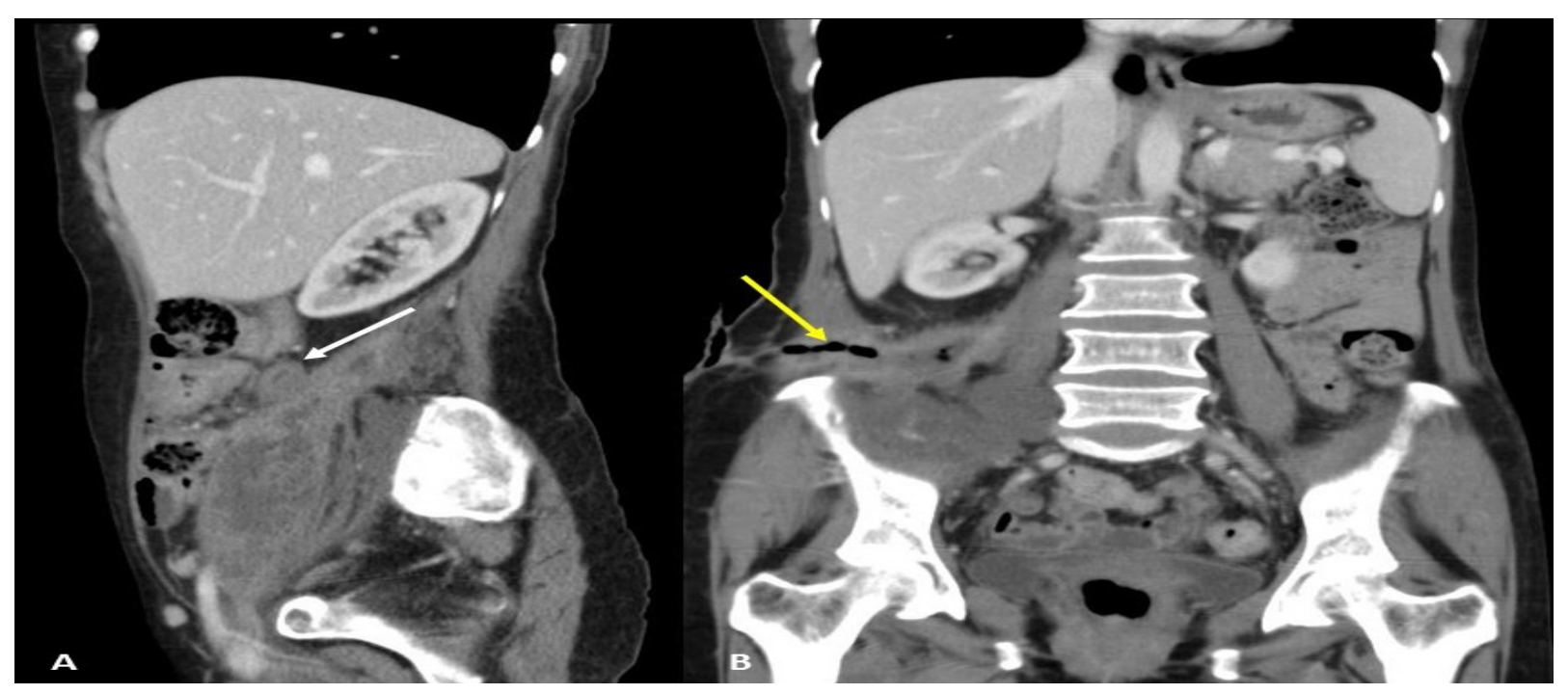

Figure 4: Contrast enhanced CT scan sagittal images (A) reveal a thick walled bowel loop closely abutting the anterosuperior margin of the retroperitoneal collection (arrow). In the coronal images (B), a fistulous tract is seen extending laterally from the retroperitoneal collection, just above the right iliac bone to the skin (yellow arrow). The visualised lumbar vertebrae and intervertebral discs appear to be uninvolved in the disease process. 


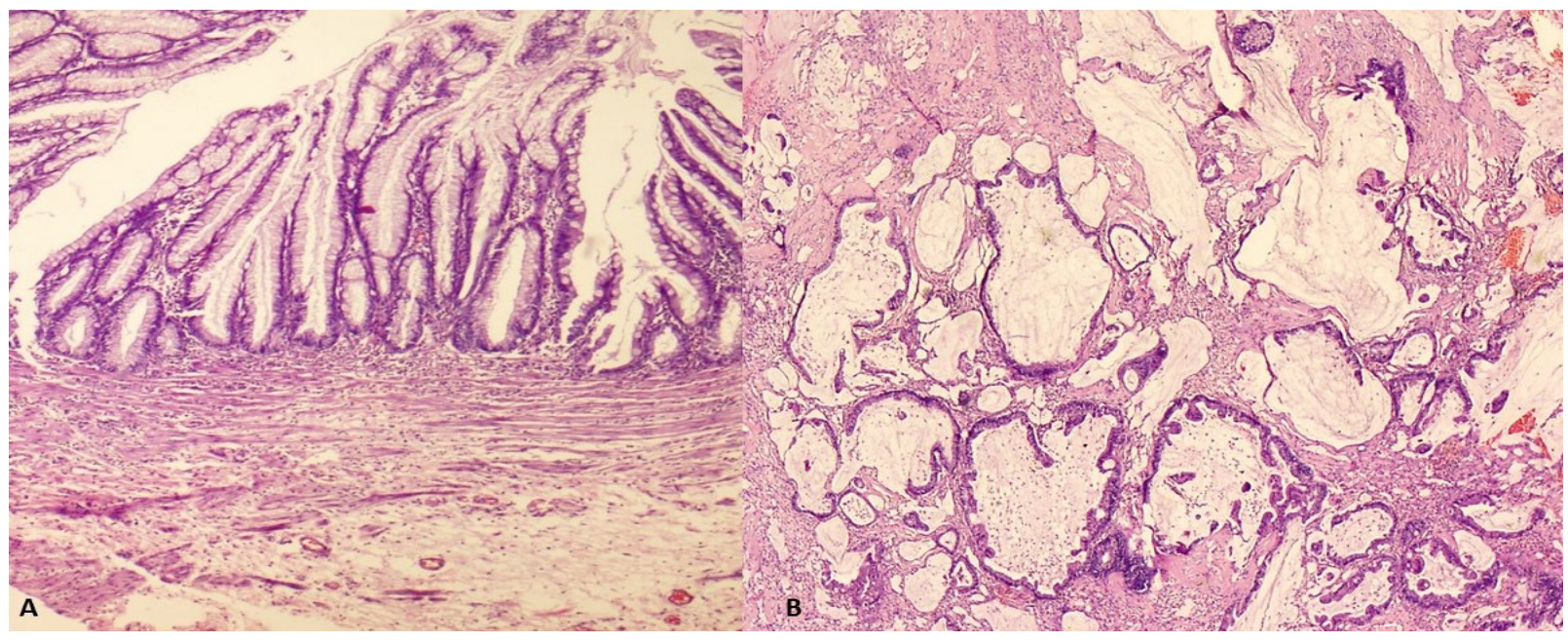

Figure 5: (A) Appendiceal cystadenoma showing extensive mucosal hyperplasia, and replacement by villous mucinous epithelium lined by goblet cells, with focal low grade dysplasia (H\&E, 20x). (B) Histopathology from the pseudomyxoma shows epithelial strips and undulating mucinous glands lined by cells with intracytoplasmic mucin, focal stratification, low grade dysplasia and occasional mitotic activity lying in pools of mucin (H\&E, 20x).

\section{Discussion}

Although pseudomyxoma peritonei and retroperitonei share a common genesis, with similar histopathological features, there is a variance in their incidence rate. Pseudomyxoma peritonei shows female predominance (female to male ratio 2-3:1) and an average age of 53 years, while PMR shows an equivocal female to male ratio of $1: 1.22$, with a maximum age incidence of 60.9 years $(6,7)$.

Literally translated, pseudomyxoma means false mucinous tumour, referring to a gelatinous or mucinous collection, commonly seen in the peritoneal cavity. Usually, the organ of origin is the appendix, with other rare primary sites being the ovaries, colon, stomach, pancreas, uterus, common bile duct and urachus $(1,6)$. Rarely, a gelatinous pseudomyxoma is confined exclusively to the retroperitoneal compartment and, accordingly, is termed pseudomyxoma retroperitonei. A retrocaecal appendix accounts for the majority of such cases, but mucinous neoplasms of the ovary, ascending colon and rectum have also been implicated in its aetiology $(1,8-10)$. The primary mucous secreting neoplasms of the appendix may range from a low grade cystadenoma to a malignant cystadenocarcinoma (4).

The retroperitoneum is a large potential space, bounded anteriorly by the posterior peritoneum, posteriorly by the spine and back muscles, superiorly by the diaphragm, inferiorly by the levators and laterally by the flank muscles. It is at the level of the anterior superior iliac spine to the tip of the twelfth rib. Thus, retroperitoneal masses can attain very large sizes before producing any signs or symptoms. In the present case, the unique retrocaecal positioning of the appendix facilitated its rupture into the retroperitoneal space, with a resultant PMR. A preoperative diagnosis of pseudomyxoma remains frustratingly elusive, since they can remain asymptomatic for long periods of time, and when symptomatic, the features are nonspecific, such as an abdominal mass, pain and weight loss $(1,4)$.

A very rare presentation of a PMR, as seen in our case, includes a spontaneous cutaneous fistulous tract opening in the right flank. Anatomically, this represents the hernial orifice of the inferior lumbar triangle (Petit's hernia), which is bounded by the external oblique muscle laterally, the latissimus dorsi medially and iliac crest inferiorly (11). Unique to our case, the PMR also extended simultaneously into the anterior thigh along the iliopsoas muscle.

Although these above mentioned clinical presentations of PMR have been described in the literature, the delay in the eventual diagnosis in our case can be ascribed to the rarity of its occurrence.

Imaging plays an important role in the diagnosis and management of PMR, and upon ultrasound and CT, it presents as a multiloculated cystic lesion showing thick walls or septae, 
displacing and distorting the adjacent structures. PMR can also appear as curvilinear or mural punctate calcifications (12). A CT scan can also help identify the primary pathology in the appendix by demonstrating its wall thickening and its retrocaecal position. The cystic character of a PMR is best demonstrated by its classic signal characteristics of T1-weighted hypointensity and T2-weighted hyperintensity upon MRI, with enhancement of its wall and thick internal septations upon post-contrast study (3). An MRI accurately maps the extent of the lesion, and helps define its relationship with adjacent structures.

The differentials for PMR include primary mucinous cystadenoma of the retroperitoneum, cystic lymphangioma, cystic teratoma, cystic degeneration of a malignant peripheral nerve sheath tumour and psoas abscess.

A primary mucinous cystadenoma presents as a unilocular cystic lesion, while a cystic lymphangioma presents as multilocular cystic lesion; however, their characteristic lack of contrast enhancement helps to easily differentiate them from PMRs. With regard to a cystic teratoma, it can be differentiated based on the fatty content, which appears to be hyperintense on both T1weighted and T2-weighted MRIs.

Heterogeneous peripheral and internal enhancement, with intratumoural cystic changes, reveals a malignant peripheral nerve sheath tumour (13). These imaging features closely mimic those of the PMR, but the close approximation of the thick walled tubular structure, extending from the ileocaecal junction to the retroperitoneal collection, clinches the diagnosis of a PMR with an appendiceal origin.

PMR has previously been documented as mimicking an iliopsoas abscess (14), which is usually associated with spondylodiscitis of the adjacent vertebrae, and shows homogenous peripheral enhancement. The lack of vertebral involvement, with morphological evidence of effacement, no invasion of the psoas muscle, and enhancing irregular internal septations within the collection, ruled out the possibility of a psoas abscess, and favoured the diagnosis of PMR.

Of the two mucin producing appendiceal neoplasms causing pseudomyxoma, adenoma/cystadenoma, due to the low grade biological activity, has a better prognosis than cystadenocarcinoma (15). Histopathologically, PMR has been classified into low and high grades based on architectural and cytomorphological patterns, which take into consideration features such as cellularity and invasiveness of the mucinous implants, complexity of the epithelial architecture, degree of cytologic atypia and the presence or absence of signet ring cells and mitosis. This classification is prognostically important, since the survival outcomes are significantly better with a low grade pathology, compared to a high grade PMR $(15,16)$.

The mainstay in the treatment of pseudomyxoma is surgical, with the goal being the removal of the primary secreting organ, and complete evacuation of the mucinous material. Adjuvant systemic chemotherapy is reserved for cases with a high pathological grade, and those where complete evacuation is not achieved (6). The reported 5-year survival of patients with pseudomyxoma peritonei ranges from $11 \%$ to $75 \%$ (mean: 50\%) (1). Due to the scarcity of reported cases, the survival rates are not well known in pseudomyxoma retroperitonei, but appear to be better than pseudomyxoma peritonei, since the disease is confined to the retroperitoneum, and the vital organs are not involved $(1,6)$.

In the present case, the histopathology revealed an appendiceal mucinous cystadenoma, with low grade dysplasia, and the pseudomyxoma was reported as a mucinous tumour of borderline potential. The patient's CA-125levels were found to be within the normal limits $(26.2 \mathrm{U} / \mathrm{ml})$, ruling out the possibility of ovarian malignancy, indicating a favourable prognosis. Since a complete surgical evacuation of the pseudomyxoma was achieved, further chemotherapy was not indicated. At the 6-month follow up, the patient was doing well, and there was no evidence of residual or recurrent tumours.

In summary, we suggest that in complaints of a non-healing sinus in the right supragluteal region, the clinician should be alert for the rare possibility of a pseudomyxoma retroperitonei. A thorough physical examination should be conducted, especially in the inguinal region, to look for extra-pelvic extensions of such retroperitoneal diseases, which can act as clues to the underlying disease. Also of importance are the imaging features of the PMR, especially upon MRI, which can help radiologists to conclusively diagnose this condition, despite its challenging differentials.

\section{Acknowledgement}

None

\section{Conflict of Interest}

None 


\section{Funds}

None

\section{Authors' Contributions}

Conception and design, Analysis and interpretation of the data, Drafting of the article, Critical revision of the article for important intellectual content, Final approval of the article: RH, SK, ACR, RK

\section{Correspondence}

Dr. Sandeep Kumar

MBBS,MD (Radiodiagnosis)

Department of Radiodiagnosis and Imaging,

Kasturba medical college, Manipal University,

Manipal, Karnataka, India. 576104

Tel: +o8202922093

Fax: -

E-mail: drsandeepkumarradiologist@gmail.com

\section{References}

1. Diaz-Zorrilla C, Ramos-De la Medina A, GrubePagola P, Ramirez-Gutierrez de Velasco A. Pseudomyxoma extraperitonei: a rare presentation of a rare tumour. Case Reports. 2013 Feb 4;2013(febo4 1):bcr2012007702-bcr2012007702.

2. Cakmak A, Karakayali F, Bayar S, Unal E, Akyol C, Kocaoğlu H. Pseudomyxoma retroperitonei presenting with a skin fistula. Turk $J$ Gastroenterol. 2009;20:79-80.

3. Koizumi J, Noguchi H. Pseudomyxoma retroperitonei with spontaneous skin fistula. Abdom Imaging. 1999;24:193-195. doi: http://dx.doi.org/10.1007/ s002619900475

4. Nakao A, Sato S, Nakashima A, et al. Appendiceal mucocele of mucinous cystadenocarcinoma with a cutaneous fistula. J Int Med Res. 2002;30:452-456. doi:http://dx.doi.org/10.1177/147323000203000416

5. Fann JI, Vierra M, Fisher D, Oberhelman HA, et al. Pseudomyxoma peritonei. Surg Gynecol Obstet. 1993;177:441-447.

6. Ioannidis O, Cheva A, Paraskevas G, Papadimitriou N, Konstantara A, Chatzopoulos S, et al. Pseudomyxoma retroperitonei: report of 2 cases and review of literature. Rev Esp Enferm Dig. 2012;104:268275. doi: http://dx.doi.org/10.4321/S113001082012000500009
7. Homeag M, Enachescu V, Pavel LE, Zaharia B, Manescu N. Pseudomyxoma peritonei. Curr Health Sci J. 2009;35:193-196.

8. Chamisa I. Pseudomyxoma extraperitonei of ovarian origin mimicking a psoas abscess. Gynecol Surg. 2011;8:373-375. doi: http://dx.doi.org/10.1007/ s10397-010-0551-x

9. Hirokawa F, Ono K, Hayashido M, et al. A case of retroperitoneal pseudomyxoma originated from mucinous adenocarcinoma of the ascending colon (in Japanese). $J$ Wakayama Med Soc 2004;55:154-158.

10. Baker WC, Goldman LB, deVere White RW. Pseudomyxoma peritonei presenting as a scrotal mass. J Urol. 1988;139:821-822.

11. Lillie GR, Deppert E. Inferior lumbar triangle hernia as a rarely reported cause of low back pain: a report of 4 cases. $J$ Chiropr Med. 2010;9:73-76. doi: http:// dx.doi.org/10.1016/j.jcm.2010.02.001

12. Yang DM, Jung DH, Kim H, Kang JH, Kim SH, Kim $\mathrm{JH}$, et al. Retroperitoneal cystic masses: CT, clinical, and pathologic findings and literature review. Radiographics. 2004;24:1353-1365. doi: http:// dx.doi.org/10.1148/rg.245045017

13. Wasa J, Nishida Y, Tsukushi S, Shido Y, Sugiura $H$, Nakashima $H$, et al. MRI features in the differentiation of malignant peripheral nerve sheath tumors and neurofibromas. AJR Am $J$ Roentgenol. 2010;194:1568-1574. doi: http://dx.doi. org/10.2214/AJR.09.2724

14. Edrees WK, Hannon RJ. Pseudomyxoma extraperitonei: a rare presentation mimicking an iliacus abscess. Ulster Med J. 1999;68:38-39.

15. Nakakura EK. Pseudomyxoma peritonei: more questions than answers. J Clin Oncol. 2012;30:24292430. doi: http://dx.doi.org/10.1200/JCO.2012.42.3 764

16. Jackson SL, Fleming RA, Loggie BW, Geisinger KR. Gelatinous ascites: a cytohistologic study of pseudomyxoma peritonei in 67 patients. Mod Pathol. 2001;14:664-671. doi: http://dx.doi.org/10.1038/ modpathol.3880370 